DE DE GRUYTER

OPEN

G

BULGARIAN ACADEMY OF SCIENCES

CYBERNETICS AND INFORMATION TECHNOLOGIES • Volume 15, No 2

Sofia • 2015

Print ISSN: 1311-9702; Online ISSN: 1314-4081

DOI: $10.1515 /$ cait-2015-0034

\title{
Comparative Study of a Super-Twisting Sliding Mode Observer and an Extended Kalman Filter for a Freeway Traffic System
}

\author{
H. Majid ${ }^{1}$, H. Abouaïssa ${ }^{2}$ \\ ${ }^{1}$ University of Sulaimani, Faculty of Engineering Sciences, Department of Civil Engineering, \\ Sulaimani, Iraq \\ ${ }^{2}$ Université Lille Nord France, F-59000 Lille, France \\ U-Artois, LGI2A, (EA. 3926) TechnoparcFutura, F-62400 Béthune, France \\ Emails:herishmm2006@gmail.com
}

Abstract: Traffic state estimation represents one of the important ingredients for traffic prediction and forecasting. The work presented in this paper deals with the estimation of traffic state variables (density and speed), using the so called SuperTwisting Sliding Mode Observer (STSM). Several numerical simulations, using simulated and real data, show the relevance of the proposed approach. In addition, a comparative study with the Extended Kalman Filter $(E K F)$ is carried-out. The comparison indices concern convergence and stability, dynamic performance and robustness. The design of the two observers is achieved using a nonlinear second order traffic flow model in the same highway traffic and geometric conditions.

Keywords: Super-twisting sliding mode observer, extended Kalman filter, traffic flow model.

\section{Introduction}

Real-Time highway traffic management and development of Intelligent Transportation Systems (ITS) remain an important area of intensive research in order to mitigate the daily problem of congestion and ensure safe and less polluting transportation of goods and people. One of the prerequisites for continuous prediction is an efficient estimation of the real-time traffic conditions, using only a limited amount of data. 
Currently, traffic data is usually obtained via a set of sensors, such as inductive loop detectors which provide occupancy rate and traffic mean speed measurements from which other macroscopic quantities can be derived when accurate vehicle length is available. These macroscopic quantities include vehicles density (number of vehicles per unit length of highway), mean speed (unit length of highway per unit of time) and flow. The high cost of installation and maintenance of a large number of sensors and the fact that the sets of data provided by the existing detectors are often incomplete and noisy, tempted many researchers to develop traffic control methods and algorithms based on state estimation techniques [1]. The main solution of such problems requires reconstruction of the missing traffic states. The works referred to state and/or parameters estimations, using sliding mode technique, are few. In K o h a n [2] proposed a robust first order sliding mode observer to estimate the traffic state variables (density and mean speed) in a freeway section. It suffered from chattering phenomena. In [3] the authors used a sliding mode observer to estimate the state variables of a second order traffic flow model in a freeway section. The authors have not found more works using a variable structure observer in highway traffic domains. On the contrary, they are well studied in the industry sector, for example, in robotics [4-6], in electrical engineering [7, 8] or in chemical reactors [9]. Notice that the widely used traffic state estimations methods are stemming from Kalman filtering techniques. In this context, in K napp [10] developed a method based on time series of speed and flow data from a set of sensors in order to generate vehicles counts. These crude estimates are then filtered using Kalman filter. In [11] it was shown how the Cell Transmission Model (CTM) can be included in the general Extended Kalman Filtering (EKF) framework, and the capability of the combined CTM-EKF model to capture (rapid) changes of important modeling parameters like capacity. In [12] an improved mixture of Kalman filter algorithm was proposed, which is based on sequential Monte Carlo method to solve the difficult problem of interference on a switching state-space model with an unobserved discrete state. In [1] the authors used a Mixture of Kalman Filtering (MKF) algorithm on the switching-mode traffic model. The estimator is able to provide the estimated vehicle densities at unmeasured locations, as well as the congestion states (free-flow or congested), which are not directly observed. In [13] a real-time estimation of the complete traffic state in freeway stretches is developed, based on an extended Kalman filter. In [14] the performance of the extended Kalman filter and the unscented Kalman filter for state estimation are compared. Generally, the extended Kalman filters are more adapted when the set of measurements and model uncertainties are assumed to be white noise with normal distributions. In practice this assumption is valid only for traffic measurements, and the uncertainties involved in the model equations, such as disturbances and modeling errors cannot be simply regarded as white noise. Moreover, several difficulties are still persistent with respect to the tuning, the numerical analysis and the sensitivity to perturbations. In [2] a short comparison between the first order sliding mode observer and EKF was achieved. Generally, the first order sliding mode observer was labeled better with respect to EKF. 
This paper focuses on the closed-loop full order observer techniques and tries to give comprehensive comparison of them, including a super-twisting sliding mode observer and an extended Kalman filter. It aims at giving a thorough evaluation of STSM observer and EKF from several aspects, including state estimation (convergence and stability), dynamic performance, parameter and noise sensitivity, and complexity. The simulation and experimental results, as well as theory analysis are presented to give a comprehensive comparison of them.

We can summarize the contributions of this paper as follows:

- Application of the most recent and robust algorithm of sliding mode technique (STSM) which is related to Variable Structure Systems (VSS) theory.

- The simulations are conducted, using real and fictive data. A control system is used to verify the stability of the two observer algorithms.

- Application of some indices in order to conduct a comparative study between the proposed algorithm and the extended Kalman Filter.

The paper is organized as follows: Section II recalls the used macroscopic model (METANET). Section III presents the design algorithms for the two observers. Section IV introduces the traffic control algorithm. Section V provides numerical simulation for a sample network. Section VI concludes the paper and outlines some tracks for future developments.

\section{Macroscopic traffic flow model}

The first macroscopic model was developed in [15] and [16] which are based on the conservation law:

$$
\frac{\partial}{\partial_{t}} \rho(x, t)+\frac{\partial}{\partial x} q(x, t)=g(x, t),
$$

where $\rho(x, t), q(x, t)$ and $g(x, t)$ are the traffic density in veh/km per lanes, the traffic volume in veh/h and the ramp generation term in veh/h per $1 \mathrm{~km}$, respectively.

Equation (1) is supplied by $(x, t)=\rho(x, t) v(x, t) \beta$, where $v(x, t)$ is the mean speed and $\beta$ is the number of lanes. The mean speed $v(x, t)$ and the traffic density $\rho(x, t)$ are related by a so called fundamental diagram, $v(x, t)=V_{\mathrm{e}}(\rho(x, t))$. One of the most widely used forms of such a function is due to M a y [17]

$$
V_{\mathrm{e}}(\rho(x, t))=v_{\mathrm{f}} \exp \left(-\frac{1}{a}\left(\frac{\rho(t)}{\rho_{\mathrm{c}}}\right)^{a}\right),
$$

where $a$ is a parameter, $v_{\mathrm{f}}$ is the free-flow speed and $\rho_{\mathrm{c}}$ represents the critical density. Such fundamental diagram allows identifying of the free flow and congested zones (Fig. 1).

Since the first order macroscopic model is based on a static relationship between the main traffic variables, it presents several drawbacks, such as the inability to describe the dynamics of the traffic behavior. In this context, the second order models seem to be more adapted. One of these models is the Payne's model [18]. In this section we present a second order traffic flow model proposed in $[19,20]$. This model represents a freeway network as a directed graph whose links are associated with a stretch in the freeway network. Each link in the graph 
corresponds to a stretch that has uniform characteristics. In the model, the $m$-th link of a freeway is divided into $n$ segments. For each link $m$ and segment $i$, the state variables of the traffic as described above are expressed as the average density, the mean speed and flow (Fig. 2). The studied freeway segment is then described by the following ordinary differential equations for each segment $i$ :

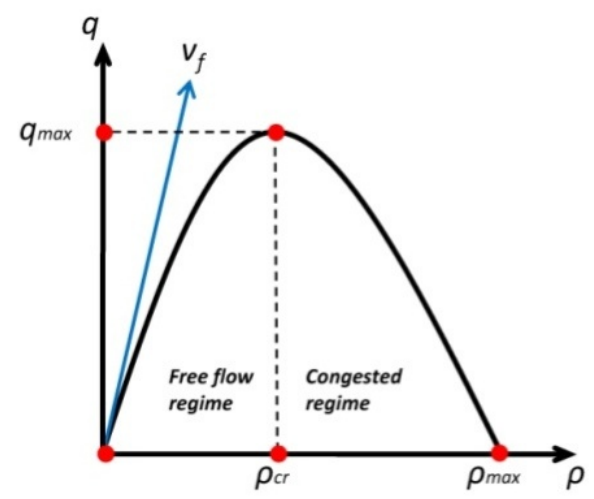

Fig. 1. Fundamental diagram

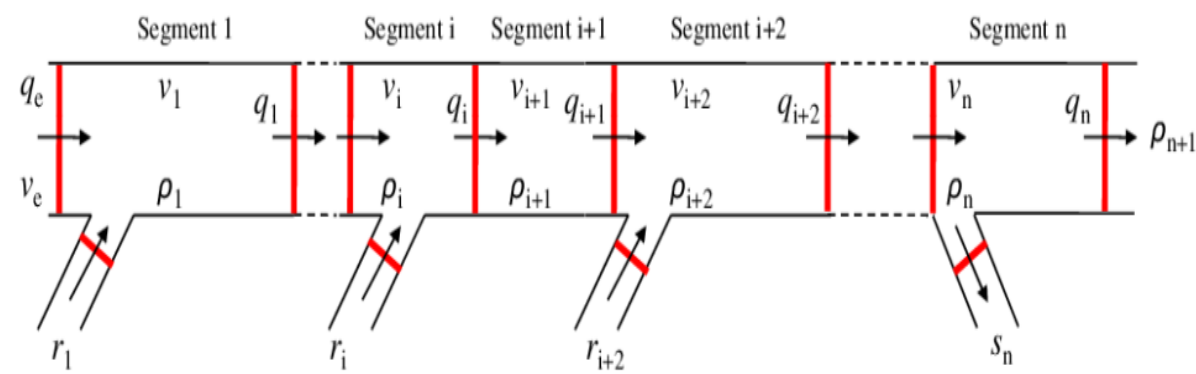

Fig. 2. Freeway example

$$
\begin{gathered}
\rho_{i}(k+1)=\rho_{i}(k)+\frac{T_{\mathrm{s}}}{L_{i}}\left(q_{i-1}(k)+r_{i}(k)-s_{i}(k)-\rho_{i}(k) v_{i}(k)\right), \\
v_{i}(k+1)=v_{i}(k)+\frac{T_{\mathrm{s}}}{\tau}\left(V_{\mathrm{e}}\left(\rho_{i}\right)(k)-v_{i}(k)\right)+ \\
+\frac{T_{\mathrm{s}}}{L_{i}} v_{i}(k)\left(v_{i-1}(k)-v_{i}(k)\right)-\frac{\eta T_{\mathrm{s}}}{\tau L_{i}} \frac{\left(\rho_{i+1}(k)-\rho_{i}(k)\right)}{\rho_{i}(k)+\kappa}-\frac{\delta q_{\mathrm{o}_{i-1}}(k) v_{i}(k)}{L_{i}\left(\rho_{i}(k)+\kappa\right)},
\end{gathered}
$$

where: $r_{i}(k)$ and $s_{i}(k)$ are the on-ramp and off-ramp flows, respectively; $\tau, \eta, \kappa$ and $\delta$ are the model parameters; $L_{i}$ is the length of the segment $i$; the last term is used if there is an on-ramp in the segment to account for the speed drop caused by the merging phenomena; $q_{\mathrm{o}_{i-1}}$ is calculated as follows [21]:

$$
q_{\mathrm{o}_{i-1}}(k)=\min \left[\begin{array}{c}
d_{\mathrm{o}_{i-1}}(k)+\frac{w_{\mathrm{o}_{i-1}}(k)}{T_{s}}, \\
Q r_{\mathrm{m}} r_{\mathrm{o}_{i}}(k), Q r_{\mathrm{m}}\left(\frac{\rho_{\mathrm{m}_{i}}-\rho_{i}}{\rho_{\mathrm{m}_{i}}-\rho_{\mathrm{c}}}\right)
\end{array}\right],
$$


where $Q r_{\mathrm{m}}$ is the on-ramp capacity, $\rho_{m}$ is the maximum (jam) density of the segment, $r_{\mathrm{o}_{i}}(k) \in[0 ; 1]$ is the on-ramp metering rate. $w_{\mathrm{o}_{i-1}}(k)$ is the queue length at segment $i-1 . w_{\mathrm{o}_{i}}(k)=0$ if $i>1, T_{\mathrm{s}}$ is the time step,

$$
w_{\mathrm{o}_{i-1}}(k+1)=w_{\mathrm{o}_{i-1}}(k)+T_{\mathrm{s}}\left(d_{\mathrm{o}_{i-1}}(k)-q_{\mathrm{o}_{i-1}}(k)\right) .
$$

\section{Observer design}

\subsection{Super-twisting sliding mode observer}

The sliding mode technique is related to the Variable Structure Systems (VSS) theory. It is essentially based on the resolution of differential equations with a discontinuous right hand side which is introduced in [22]. Historically, the twisting mode algorithm is the first 2nd order sliding mode algorithm known [23]. It features are twisting around the origin of the 2nd order sliding plane. The trajectories perform an infinite number of rotations while converging in finite time to the origin [24]. In the super-twisting algorithm the trajectories on the 2nd order sliding plane are also characterized by twisting around the origin, see Fig. 3.

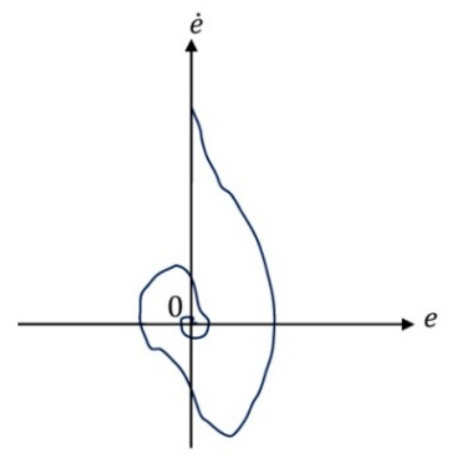

Fig. 3. Super-twisting algorithm phase trajectory

\subsubsection{Short overview of the observer}

The sliding mode technique has been used for the observer's design in many applications [25-27]. Let us consider a SISO nonlinear system:

$$
\left\{\begin{array}{c}
\dot{x}_{1}=x_{2} \\
\dot{x}_{2}=x_{3} \\
\vdots \\
\dot{x}_{n-1}=x_{n} \\
\dot{x}_{n}=f\left(x_{1}, \ldots, x_{n}\right) \\
y=x_{1}
\end{array}\right.
$$

where $x=\left[x_{1}, \ldots, x_{n}\right]^{\mathrm{T}} \in \mathcal{R}^{n}$ is the state vector, $y=x_{1} \in \mathcal{R}$ is the output vector. The super twisting algorithm based on the step-by-step sliding mode observers is designed as follows: 


$$
\left\{\begin{array}{c}
\dot{\hat{x}}_{1}=\tilde{x}_{2}+\lambda_{1}\left|e_{1}\right|^{\frac{1}{2}} \operatorname{sign}\left(e_{1}\right) \\
\dot{\tilde{x}}_{2}=\pi_{1} \operatorname{sign}\left(e_{1}\right) \\
\dot{\hat{x}}_{2}=E_{1}\left[\tilde{x}_{3}+\lambda_{2}\left|e_{2}\right|^{\frac{1}{2}} \operatorname{sign}\left(e_{2}\right)\right] \\
\dot{\tilde{x}}_{3}=E_{1} \pi_{2} \operatorname{sign}\left(e_{2}\right) \\
\dot{\hat{x}}_{3}=E_{2}\left[\tilde{x}_{4}+\lambda_{3}\left|e_{3}\right|^{\frac{1}{2}} \operatorname{sign}\left(e_{3}\right)\right] \\
\vdots \\
\dot{\tilde{x}}_{n-1}=E_{n-3} \pi_{n-2} \operatorname{sign}\left(e_{n-2}\right) \\
\dot{\hat{x}}_{n-1}=E_{n-2}\left[\tilde{x}_{n}+\lambda_{n-1}\left|e_{n-1}\right|^{\frac{1}{2}} \operatorname{sign}\left(e_{n-1}\right)\right] \\
\dot{\tilde{x}}_{n}=E_{n-2} \pi_{n-1} \operatorname{sign}\left(e_{n-1}\right) \\
\dot{\hat{x}}_{n}=E_{n-1}\left[\tilde{\theta}+\lambda_{n}\left|e_{n}\right|^{\frac{1}{2}} \operatorname{sign}\left(e_{n}\right)\right] \\
\tilde{\theta}=E_{n-1} \pi_{n} \operatorname{sign}\left(e_{n}\right)
\end{array}\right.
$$

where $e_{i}=\tilde{x}_{i}-\hat{x}_{i}$ for $i=1, \ldots, n$, with $\tilde{x}_{1}=x_{1}$ and $[\tilde{x}, \tilde{\theta}]^{\mathrm{T}}=\left[\tilde{x}_{1}, \tilde{x}_{2}, \ldots, \tilde{x}_{n}, \tilde{\theta}\right]^{\mathrm{T}}$ is the output of the observer. For $i=1, \ldots, n-1$, the scalar functions $E_{i}$ is defined as: $E_{i}=1$ if $\left|e_{j}\right|=\left|\tilde{x}_{j}-\hat{x}_{j}\right| \leq \varepsilon$, for all $j \leq i$ else $E_{i}=0$; where $\varepsilon$ is a small positive constant. The observer gains $\lambda_{i}$ and $\pi_{i}$ are positive scalars. The convergence of the state observation error is obtained in $n-1$ steps and in finite time. The sliding mode differentiator of order 2 (super twisting algorithm) is given in its general form by [28] (Fig. 4).

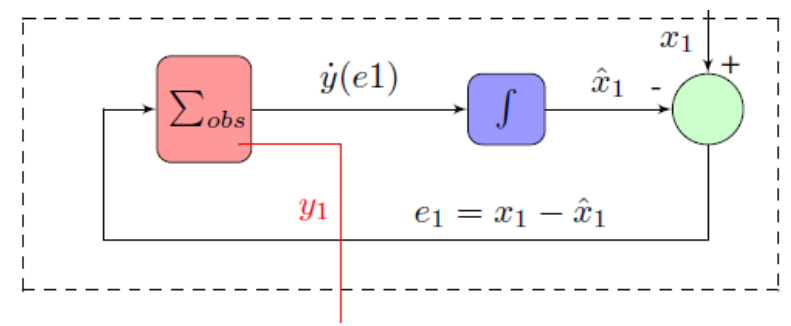

Fig. 4. Structure of the super twisting algorithm

$$
\sum_{\mathrm{obs}}=\left\{\begin{array}{c}
\dot{y}\left(e_{1}\right)=y_{1}+\lambda_{1}\left|e_{1}\right|^{\frac{1}{2}} \operatorname{sign}\left(e_{1}\right) \\
\dot{y}_{1}=\pi_{1} \operatorname{sign}\left(e_{1}\right)
\end{array}\right\}, \lambda_{1}, \pi_{1}>0,
$$

where, $\lambda_{1}$ and $\pi_{1}$ are positive tuning parameters of the differentiator whose output is $y$, and $y_{1}$ is the output of the observer. Applying the exact differentiator to system (7) when $n=2$, one obtains:

$$
\begin{gathered}
\dot{\hat{x}}_{1}=\tilde{x}_{2}+\lambda_{1}\left|e_{1}\right|^{\frac{1}{2}} \operatorname{sign}\left(e_{1}\right), \\
\dot{\tilde{x}}_{2}=\pi_{1} \operatorname{sign}\left(e_{1}\right), \\
\dot{\hat{x}}_{2}=\tilde{\theta}+\lambda_{2}\left|e_{2}\right|^{\frac{1}{2}} \operatorname{sign}\left(e_{2}\right), \\
\dot{\tilde{\theta}}=\pi_{2} \operatorname{sign}\left(e_{2}\right) .
\end{gathered}
$$


The convergence of the observation error is obtained in one step in finite time. Another feature of the differentiators, Equation (8), is the fact that the output does not depend directly on discontinuous functions but on an integrator output. Therefore, high frequency chattering is attenuated [29]. Both properties are important, since the switching function can be obtained in a continuous way without delays [30]. See [26, 27, 30-34] for more details and discussions.

\subsubsection{Traffic state estimation}

Consider the following freeway segments shown in Fig. 5. Using the relationship between the three aggregated variables (flow $q$, density $\rho$ (For simulation purpose, the occupancy rate $T_{\mathrm{o}}$ which is provided by the loop-detector, is transformed in term of traffic density thanks to the following expression: $T_{0}=100\left(L_{\mathrm{b}}+L_{\mathrm{v}}\right) \rho$ [35]. $L_{\mathrm{b}}$ and $L_{\mathrm{V}}$ are the lengths of the loop detector and the vehicle length respectively.) and the mean speed

$$
v: q_{i}(x, t)=\rho_{i}(x, t) v_{i}(x, t) .
$$

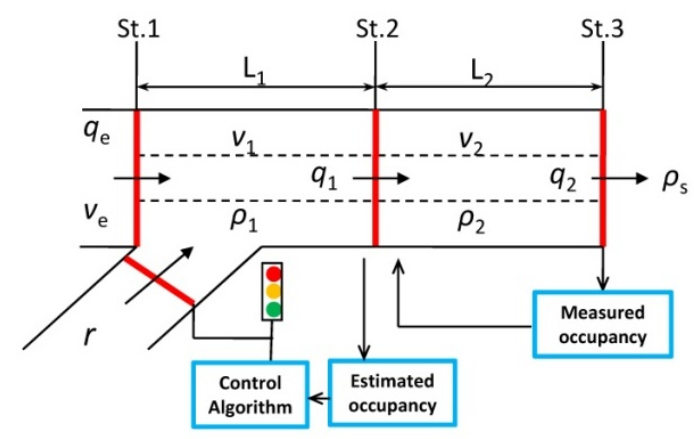

Fig. 5. Freeway segments example

The output vector is $y=\left[\begin{array}{ll}y_{1} & y_{2}\end{array}\right]^{\mathrm{T}}=\left[\begin{array}{ll}\rho_{2} & v_{2}\end{array}\right]^{\mathrm{T}}$. The studied freeway section (Fig. 5) can be described by the following equations:

$$
\begin{gathered}
\rho_{1}(k+1)=\rho_{1}(k)+\frac{T_{\mathrm{s}}}{L_{1}}\left(q_{\mathrm{e}}(k)+r(k)-\rho_{1}(k) v_{1}(k)\right), \\
\rho_{2}(k+1)=\rho_{2}(k)+\frac{T_{\mathrm{s}}}{L_{2}}\left(\rho_{1}(k) v_{1}(k)-\rho_{2}(k) v_{2}(k)\right), \\
v_{1}(k+1)=v_{1}(k)+\frac{T_{\mathrm{s}}}{\tau}\left(V_{\mathrm{e}}\left(\rho_{1}\right)(k)-v_{1}(k)\right)+ \\
+\frac{T_{\mathrm{s}}}{L_{1}} v_{1}(k)\left(v_{\mathrm{e}}(k)-v_{1}(k)\right)-\frac{\eta T_{\mathrm{s}}}{\tau L_{1}} \frac{\left(\rho_{2}(k)-\rho_{1}(k)\right)}{\rho_{1}(k)+\kappa}-\frac{\delta q_{\mathrm{o}}(k) v_{1}(k)}{L_{1}\left(\rho_{1}(k)+\kappa\right)}, \\
v_{2}(k+1)=v_{2}(k)+\frac{T_{\mathrm{s}}}{\tau}\left(V_{\mathrm{e}}\left(\rho_{2}\right)(k)-v_{2}(k)\right)+ \\
+\frac{T_{\mathrm{s}}}{L_{1}} v_{2}(k)\left(v_{1}(k)-v_{2}(k)\right)-\frac{\eta T_{\mathrm{s}}}{\tau L_{2}} \frac{\left(\rho_{s}(k)-\rho_{2}(k)\right)}{\rho_{2}(k)+\kappa}
\end{gathered}
$$

where: $\rho_{2}(k)$ and $v_{2}(k)$ are assumed to be known; $V_{\mathrm{e}}(k) \rho_{i}(k)$ are given thanks to May's fundamental diagram; $\rho_{\mathrm{s}}(k)$ is the density of the last segment, if $\rho_{2}(k)<\rho_{\mathrm{c}}$ then $\rho_{\mathrm{s}}(k)=\rho_{2}(k)$, if not $\rho_{\mathrm{s}}(k)=\rho_{\mathrm{c}} ; q_{2}(k)$ and $v_{2}(k)$ are state variables measured thanks to the loop detector located at St. 3; $q_{\mathrm{e}}(k)$ and $v_{\mathrm{e}}(k)$ (measured at 
sensor St. 1) are external measured inputs (Fig. 5). Let us assume that the loop detector located at St. 2 is broken down. The main objective is then to estimate the state variables $\rho_{1}(k)$ and $v_{1}(k)$ which are of important use for the control system from the measured states provided by station St. 3 .

Let $\tilde{\rho}(k), \hat{\rho}(k)$ and $\tilde{v}(k), \hat{v}(k)$ are the estimated values of $\rho_{1}(k), \rho_{2}(k)$, and $v_{1}(k), v_{2}(k)$ respectively. Then the observer errors are defined as: $e_{\rho}(k)=\rho(k)-$ $\hat{\rho}(k)$ and $e_{v}(k)=v(k)-\hat{v}(k)$. The proposed observer (called super-twisting) has the form

(18) $\hat{\rho}_{2}(k+1)=\hat{\rho}_{2}(k)+\frac{T_{\mathrm{s}}}{L_{2}}\left(\tilde{\rho}_{1}(k) \tilde{v}_{1}(k)-\rho_{2}(k) v_{2}(k)\right)+z_{\rho_{2}}(k)$, where $\tilde{\rho}_{1}(k)$ and $z_{\rho_{2}}(k)$ are of the form:

$$
\begin{gathered}
\tilde{\rho}_{1}(k)=\pi_{\rho}(k) \operatorname{sign}\left(\rho_{2}(k)-\hat{\rho}_{2}(k)\right), \\
z_{\rho_{2}}(k)=\lambda_{\rho(k)}\left|\rho_{2}(k)-\hat{\rho}_{2}(k)\right|^{\frac{1}{2}} \operatorname{sign}\left(\rho_{2}(k)-\hat{\rho}_{2}(k)\right) .
\end{gathered}
$$

For the speed estimation $\tilde{v}_{1}(k), \hat{v}_{2}(k)$ the observer reads:

$$
\begin{gathered}
\hat{v}_{2}(k+1)=\hat{v}_{2}(k)+\frac{T_{\mathrm{s}}}{\tau}\left(V_{\mathrm{e}}\left(\rho_{2}\right)(k)-\hat{v}_{2}(k)\right)+ \\
+\frac{T_{\mathrm{s}}}{L_{1}} \hat{v}_{2}(k)\left(\tilde{v}_{1}(k)-\hat{v}_{2}(k)\right)-\frac{\eta T_{\mathrm{s}}}{\tau L_{2}} \frac{\left(\rho_{\mathrm{s}}(k)-\rho_{2}(k)\right)}{\rho_{2}(k)+\kappa}+z_{v_{2}}(k), \\
\tilde{v}_{1}(k)=\pi_{v}(k) \operatorname{sign}\left(v_{2}(k)-\hat{v}_{2}(k)\right), \\
z_{v_{2}}(k)=\lambda_{v(k)}\left|v_{2}(k)-\hat{v}_{2}(k)\right|^{\frac{1}{2}} \operatorname{sign}\left(v_{2}(k)-\hat{v}_{2}(k)\right) .
\end{gathered}
$$

\subsection{Extended Kalman filter}

The Kalman filter is a recursive state estimator capable of use of multi-input, multioutput systems with noisy measurement data and process noise. It uses the plant's input and output measurements together with a state-space model of the system to give optimal estimation of the system states. The filter is optimal in the sense that the state estimates are based on a performance criterion that minimizes the meansquare error, defined as the difference between the actual and estimated states. The extended Kalman filter is a direct extension of the standard Kalman algorithm to the nonlinear system case. In particular, the Kalman gain $K$ is computed on the basis of the linearized system at the estimated state $\widehat{X}$ [36]. In our case, EKF is used to estimate the state variables (the mean speed and density) of the traffic flow simulation results.

\subsubsection{Short overview on the observer}

EKF algorithm is shown in Fig. 6. The state prediction, state covariance prediction, Kalman gain calculation, state covariance update and state estimation update are given in the next equations: 


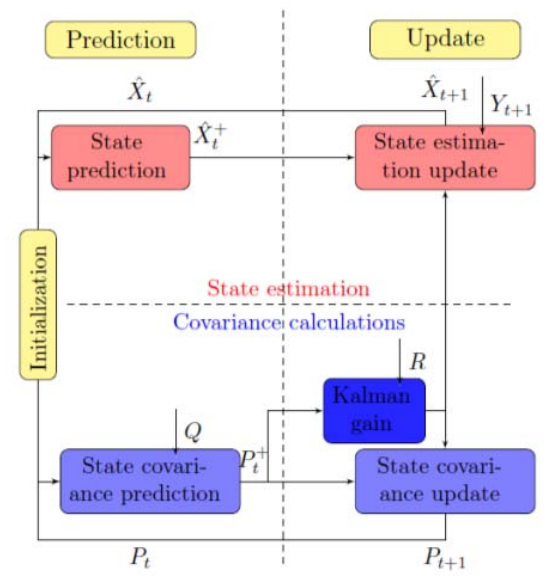

Fig. 6. EKF algorithm

$$
\begin{gathered}
\hat{X}_{k}^{+}=f\left(\hat{X}_{k}\right), \\
P_{k}^{+}=A P_{k} A^{\mathrm{T}}+Q, \\
K_{k+1}=P_{k}^{+} H_{k+1}^{T}\left(R_{k+1}+H_{k+1} P_{k}^{+} H_{k+1}^{\mathrm{T}}\right)^{-1}, \\
P_{k+1}=\left(I-K_{k+1} H_{k+1}\right) P_{k}^{+}, \\
\hat{X}_{k+1}=\hat{X}_{k}^{+}+K_{k+1}\left(Y_{k+1}-h\left(\hat{X}_{k}^{+}, 0\right)\right),
\end{gathered}
$$

where $H$ is the observation matrix, $A$ is the transition matrix, both are Jacobian matrices; $h$ is the matrix of estimated values for the measured variables; $f$ is the matrix of state equations; $R$ is the measurement noise covariance; $Q$ is the process noise covariance; $K$ is the Kalman gain, and $P$ is the estimation error covariance;

$$
\begin{gathered}
H_{k}=\frac{\partial h}{\partial X} \mid \hat{X}_{k}^{+}, \\
A_{k}=\frac{\partial f}{\partial X} \mid \hat{X}_{k-1} .
\end{gathered}
$$

\subsubsection{Traffic state estimation}

Herein we consider the same freeway segments shown in Fig. 5. It is the same section used by the Super-Twisting Sliding Mode (STSM) observer which let us compare these two observers. At first, the initial condition for the state variables $\hat{X}(0)$ and for $P(0)$ matrix must be introduced. According to the quantity of the noise in the measurement and process data, $R$ and $Q$ should be tuned, respectively, to produce the satisfied result,

$$
\begin{aligned}
& \hat{X}(0)=\left[\begin{array}{llll}
\rho_{1}(0) & v_{1}(0) & \rho_{2}(0) & v_{2}(0)
\end{array}\right]^{\mathrm{T}},
\end{aligned}
$$

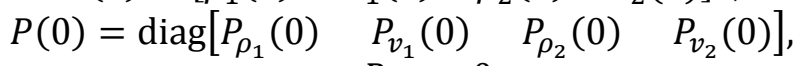

$$
\begin{aligned}
& R=\left[\begin{array}{cc}
R_{\rho_{2}} & 0 \\
0 & R_{v_{2}}
\end{array}\right],
\end{aligned}
$$




$$
Q=\left[\begin{array}{cccc}
Q_{\rho_{1}} & 0 & 0 & 0 \\
0 & Q_{v_{1}} & 0 & 0 \\
0 & 0 & Q_{\rho_{2}} & 0 \\
0 & 0 & 0 & Q_{v_{2}}
\end{array}\right],
$$

$A$ and $H$ are Jacobian matrices, constructed as follows:

$$
\begin{gathered}
A=\left[\begin{array}{llll}
\frac{\partial \rho_{1}}{\partial \rho_{1}} & \frac{\partial \rho_{1}}{\partial v_{1}} & \frac{\partial \rho_{1}}{\partial \rho_{2}} & \frac{\partial \rho_{1}}{\partial v_{2}} \\
\frac{\partial v_{1}}{\partial \rho_{1}} & \frac{\partial v_{1}}{\partial v_{1}} & \frac{\partial v_{1}}{\partial \rho_{2}} & \frac{\partial v_{1}}{\partial v_{2}} \\
\frac{\partial \rho_{2}}{\partial \rho_{1}} & \frac{\partial \rho_{2}}{\partial v_{1}} & \frac{\partial \rho_{2}}{\partial \rho_{2}} & \frac{\partial \rho_{2}}{\partial v_{2}} \\
\frac{\partial v_{2}}{\partial \rho_{1}} & \frac{\partial v_{2}}{\partial v_{1}} & \frac{\partial v_{2}}{\partial \rho_{2}} & \frac{\partial v_{2}}{\partial v_{2}}
\end{array}\right], \\
H=\left[\begin{array}{cccc}
0 & 0 & \frac{\partial h}{\partial \rho_{2}} & 0 \\
0 & 0 & 0 & \frac{\partial h}{\partial v_{2}}
\end{array}\right],
\end{gathered}
$$

where

and

$$
\begin{gathered}
\rho_{1}(k+1)=\rho_{1}(k)+\frac{T_{\mathrm{s}}}{L_{1}}\left(q_{\mathrm{e}}(k)+r(k)-\rho_{1}(k) v_{1}(k)\right) \\
\rho_{2}(k+1)=\rho_{2}(k)+\frac{T_{\mathrm{s}}}{L_{2}}\left(\rho_{1}(k) v_{1}(k)-\rho_{2}(k) v_{2}(k)\right), \\
v_{1}(k+1)=v_{1}(k)+\frac{T_{\mathrm{s}}}{\tau}\left(V_{\mathrm{e}}\left(\rho_{1}\right)(k)-v_{1}(k)\right)+ \\
+\frac{T_{\mathrm{s}}}{L_{1}} v_{1}(k)\left(v_{\mathrm{e}}(k)-v_{1}(k)\right)-\frac{\eta T_{\mathrm{s}}}{\tau L_{1}} \frac{\left(\rho_{2}(k)-\rho_{1}(k)\right)}{\rho_{1}(k)+\kappa}-\frac{\delta q_{\mathrm{o}}(k) v_{1}(k)}{L_{1}\left(\rho_{1}(k)+\kappa\right)^{\prime}} \\
v_{2}(k+1)=v_{2}(k)+\frac{T_{\mathrm{s}}}{\tau}\left(V_{\mathrm{e}}\left(\rho_{2}\right)(k)-v_{2}(k)\right)+ \\
+\frac{T_{\mathrm{s}}}{L_{1}} v_{2}(k)\left(v_{1}(k)-v_{2}(k)\right)-\frac{\eta T_{\mathrm{s}}}{\tau L_{2}} \frac{\left(\rho_{\mathrm{s}}(k)-\rho_{2}(k)\right)}{\rho_{2}(k)+\kappa}
\end{gathered}
$$

$$
\begin{gathered}
\hat{X}_{k}=\left[\begin{array}{llll}
\rho_{1}(k) & v_{1}(k) & \rho_{2}(k) & v_{2}(k)
\end{array}\right]^{\mathrm{T}}, \\
\hat{X}_{k}^{+}=\left[\begin{array}{llll}
\rho_{1}(k+1) & v_{1}(k+1) & \rho_{2}(k+1) & v_{2}(k+1)
\end{array}\right]^{\mathrm{T}} .
\end{gathered}
$$

\section{Simulation results}

For the comparison studies of the two observer (Super-twisting sliding mode STSM and extended Kalman filter EKF), the theoretical results are illustrated by some simulations. We consider the same example of the stretch depicted in Fig. 5, and we assume that the loop detector at St. 2 is broken down. Thus, in order the control system not be disrupted, the state variables $\left(\rho_{1}\right.$ and $v_{1}$ ) have been estimated from the measured state variables $\left(\rho_{2}\right.$ and $\left.v_{2}\right)$ at St. 3. The origin and ramp traffic flow used in the simulation are shown in Fig. 7. The model parameters are given in 
Table 1. For the simulation purpose, MATLAB programming language and Simulink have been used.

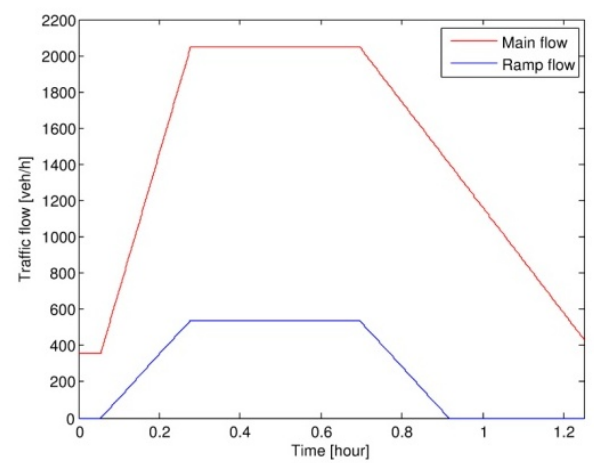

Fig. 7. Origin and ramp traffic flow used in the simulation: Main flow (upper line) and Ramp flow (low line)

Table 1. Parameters of the model

\begin{tabular}{|l|l|l|l|}
\hline Parameter & Value & Parameter & Value \\
\hline Free-flow speed & $112 \mathrm{~km} / \mathrm{h}$ & $\eta$ & 0.2 \\
\hline Jam density & $180 \mathrm{veh} / \mathrm{km}$ & $\kappa$ & $35 \mathrm{veh} / \mathrm{km}$ \\
\hline Critical density & $35.86 \mathrm{veh} / \mathrm{km}$ & $a$ & 1.40 \\
\hline$L_{1}, L_{2}$ & $100 \mathrm{~m}$ & $\tau$ & $28.08 \mathrm{~s}$ \\
\hline$\delta$ & $0.17 \mathrm{~m} / \mathrm{veh}$ & \multicolumn{2}{|l}{} \\
\cline { 1 - 3 } & & &
\end{tabular}

As a control algorithm, ALINEA is used. It is a first feedback control used in freeway traffic control [37, 38]. It is very well known by the highway traffic community. Here, ALINEA is only used to check the stability and convergence of the two observers (STSM and EKF).

\subsection{Convergence and stability}

In this section there is no parameter uncertainty. It is supposed that the model reflects $100 \%$ the reality and no external noise is present. The STSM observer gains are $\pi_{v}=0.2, \pi_{\rho}=2.2 \times 10^{-4}, \lambda_{v}=9 \times 10^{-3}$ and $\lambda_{\rho}=10^{-2}$, and $Q, R, \hat{x}$ and $P(0)$ matrices of EKF are set as:

$$
\begin{gathered}
Q=\operatorname{diag}\left[\begin{array}{llll}
10^{-4} & 10^{-4} & 10^{-1} & 10^{-1}
\end{array}\right], R=10^{-6} \operatorname{diag}\left[\begin{array}{lll}
1 & 1
\end{array}\right], \\
\hat{x}(0)=\left[\begin{array}{lllll}
8 \times 10^{-3} & 10.4 & 8 \times 10^{-3} & 10.4
\end{array}\right]^{\mathrm{T}}, \\
P(0)=\operatorname{diag}\left[\begin{array}{llll}
10^{-4} & 10^{-4} & 10^{-4} & 10^{-4}
\end{array}\right] .
\end{gathered}
$$

In this section, in order to verify the degree of convergence of the two observers, (the initial conditions of the state variables $v$ and $\rho$ of the model are different with respect to the initial conditions of the state variables $v$ and $\rho$ ) of the observers. For the model $\rho_{\text {in }}=10 \mathrm{veh} / \mathrm{km}$ and $v_{\text {in }}=46.8 \mathrm{~km} / \mathrm{h}$. For the two observers $\rho_{\text {in }}=8 \mathrm{veh} / \mathrm{km}$ and $v_{\text {in }}=37.44 \mathrm{~km} / \mathrm{h}$. Fig. 8 shows a comparison between the control value using the simulated data and the control value using STSM and EKF, respectively.

From this figure we can see that both observers converge rapidly and the control values are quite stable and can steer the system without difficulty. 
Generally, one can note that the system with EKF is considerably more stable and converges better with respect to STSM.
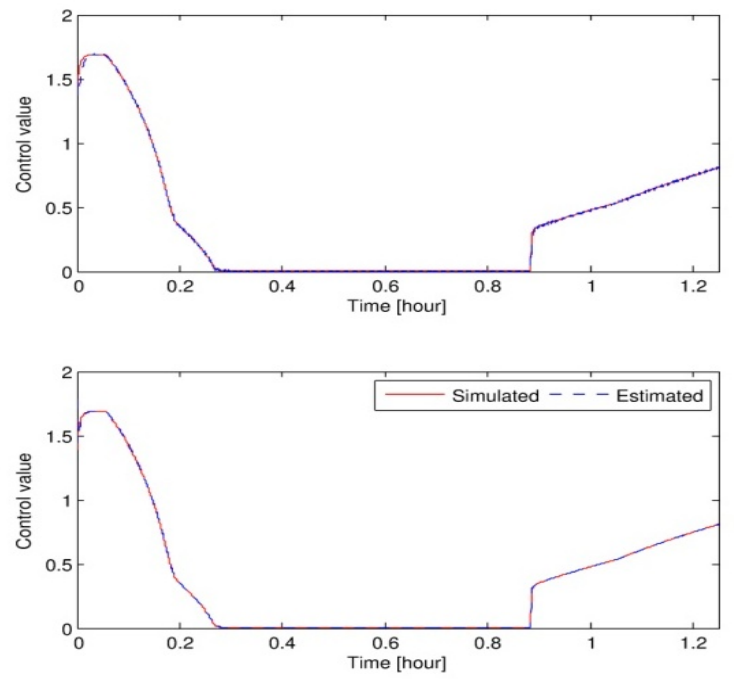

Fig. 8. Comparison of the control value using: STSM observer and simulated values (top), EKF and simulated values (bottom)

From these results, the convergence and stability of the two types of observers in terms of density and mean speed are reasonably similar under the ideal conditions of no model and parameter uncertainty, and no measurement noise (Figs 9 and 10).
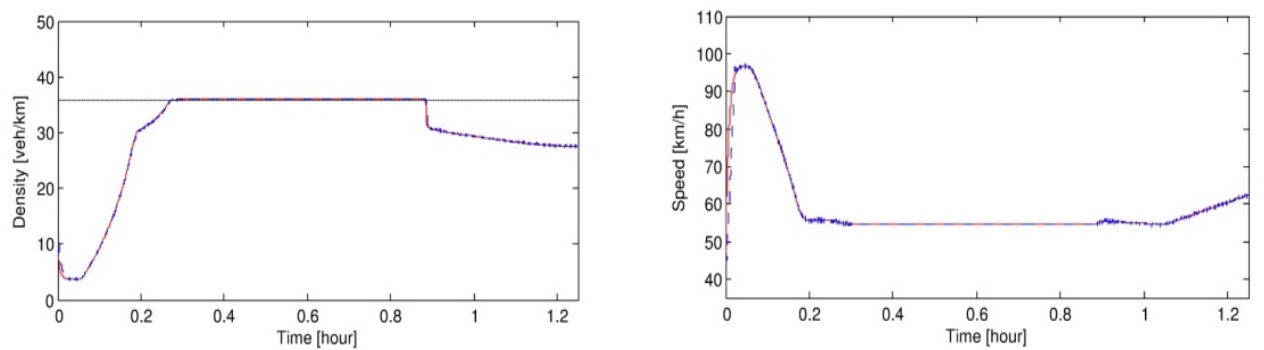

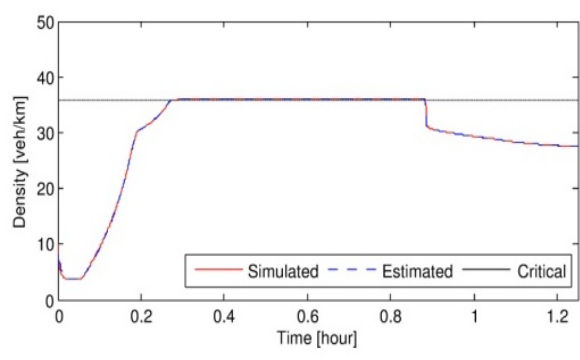

Fig. 9. Section 1: Simulated and estimated density, using STSM (top); simulated and estimated density, using EKF (bottom)

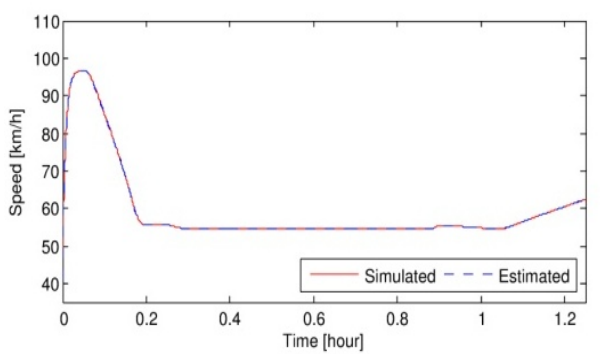

Fig. 10. Section 1: Simulated and estimated mean speed, using STSM (top); simulated and estimated mean speed, using EKF (bottom) 


\subsection{Performance}

To investigate the efficiency of the proposed STSM observer and compare it to $\mathrm{EKF}$, it proves to be more informative to perform a number of experimental scenarios and verify the observers performance in real-life conditions rather than present simulation results. For this purpose we considered data collected on the M42 motorway in the United Kingdom. The identified values for the parameters of the system, given in Table 1, are used in the implementation of the observers. The STSM observer gains are $\pi_{v}=0.7, \pi_{\rho}=10^{-3}, \lambda_{v}=0.1, \lambda_{\rho}=1.7 \times 10^{-2}$. For EKF, $R$ and $Q$ matrices were tuned to minimize the estimated error. It is initialized at $\hat{x}$ and $P(0)$ for all experiments;

$$
Q=10^{-3} \operatorname{diag}\left[10^{-3} \quad 1 \quad 1 \quad 1\right], \quad R=10^{-4} \operatorname{diag}\left[\begin{array}{ll}
0.1 & 10
\end{array}\right],
$$

$\hat{x}(0)=\left[\begin{array}{llll}2.5 & 96 & 2.5 & 96\end{array}\right]^{\mathrm{T}}, \quad P(0)=\operatorname{diag}\left[\begin{array}{llll}10^{-4} & 10^{-4} & 10^{-4} & 10^{-4}\end{array}\right]$.

Figs 11-14 show the estimation of the density and mean speed using the STSM observer and the EKF, as well as the measured data from the detector stations 7 and 8, for the period between 6 a.m.-22 p.m. in October and November of 2008. Both estimators describe the traffic density and mean speed with satisfactory accuracy. EKF makes better estimations of the density and mean speed than STSM in both stations.
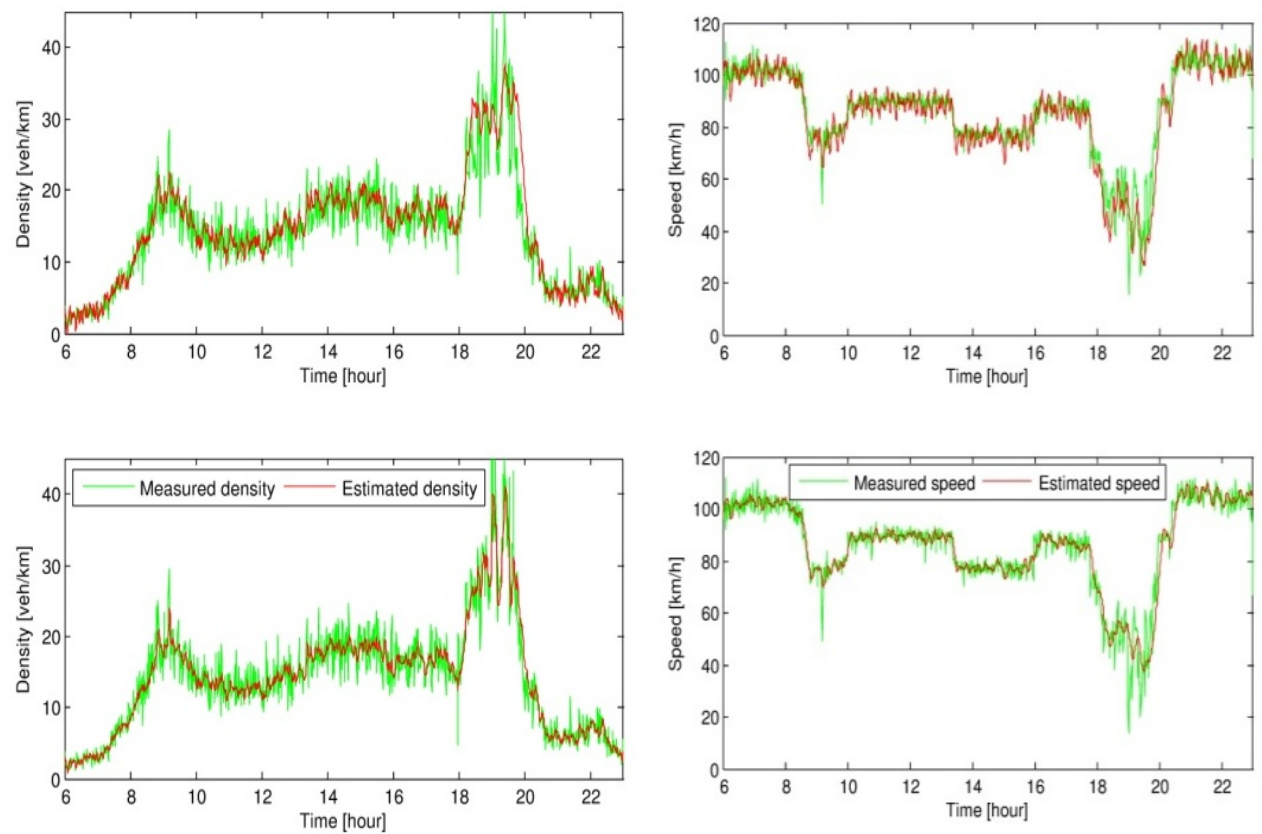

Fig. 11. Measured and estimated density using STSM. Section 1 (top), section 2 (bottom)

Fig. 12. Measured and estimated mean speed using STSM. Section 1 (top), section 2 (bottom) 

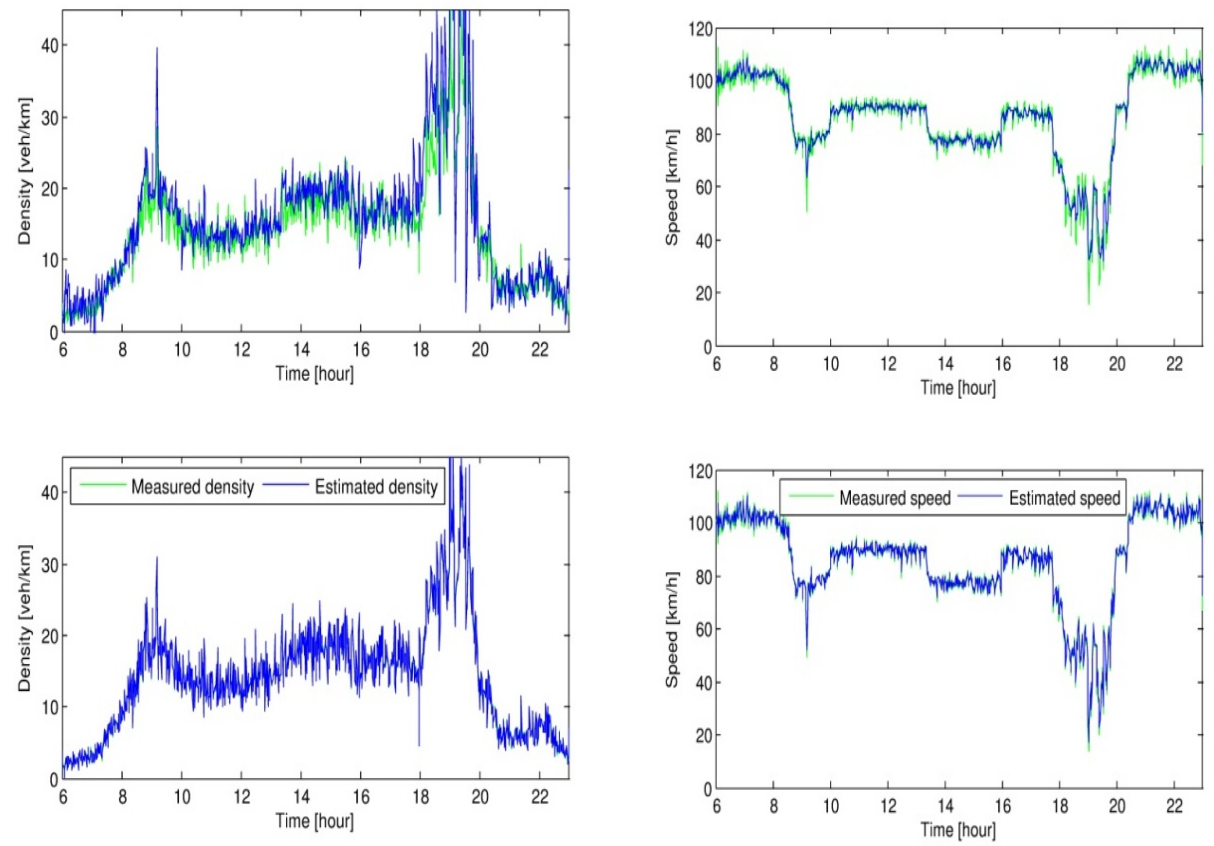

Fig. 13. Measured and estimated density using EKF. Section 1 (top), section 2 (bottom)

Fig. 14. Measured and estimated mean speed using EKF. Section 1 (top), section 2 (bottom)

The accuracy of EKF's performance depends significantly on the accuracy of $Q$ and $R$ matrices, as these matrices determine the Kalman gain $K_{f}$ and the final value of the estimation error covariance matrix. It must be noted that the time step of the real data is 6 minutes which is relatively large. The accuracy of STSM can be improved by decreasing the step time. Thus, if the time step is reduced, a better result of STSM can be achieved. To quantify the difference in the performance, we computed RMSD [39] for the estimated errors in density and the mean speed at stations 7 and 8, which is a good measure of accuracy, defined as

$$
\operatorname{RMSD}=\sqrt{\frac{\sum_{k=1}^{n}\left(x_{k}-\hat{x}_{k}\right)^{2}}{n}},
$$

where: RMSD is the square root of the mean of the squares of the deviations; $\hat{x}_{k}$ is the estimated value of the variable $x$ for time $k ; x$ is the measured value; $n$ is the number of samples. Table 2 summarizes these results. While both estimators show similar results, EKF provides smaller values for $\mathrm{RSMD}_{v}$ and $\mathrm{RSMD}_{\rho}$ than for STSM.

Table 2. RMSD of density and mean speed for the two estimator

\begin{tabular}{|c|c|c|}
\hline Estimator & STSM & EKF \\
\hline$v_{1}$ & 5.46 & 2.84 \\
\hline$v_{2}$ & 4.74 & 2.5 \\
\hline$\rho_{1}$ & 4 & 4.2 \\
\hline$\rho_{2}$ & 3.7 & 2 \\
\hline \multicolumn{2}{|c|}{$\rho$ in veh $/ \mathrm{km}, v$ in $\mathrm{km} / \mathrm{h}$} \\
\hline
\end{tabular}




\subsection{Parameter sensitivity}

Our model is time-varying. The values of its parameters cannot be fixed exactly. Therefore, there is uncertainty with respect to the parameters and the accuracy of the model. Here, to investigate the robustness, the sensitivity of the two estimators with respect to the parameter variations is found. Consider the cost function

$$
U(\varnothing)=U_{v}(\varnothing)+\frac{\sigma_{v}}{\sigma_{\rho}} U_{\rho}(\varnothing),
$$

where $\varnothing=\left[\begin{array}{lllllll}a & \tau & \eta & v_{f} & \rho_{c} & \kappa & \delta\end{array}\right], U_{v}$ and $U_{\rho}$ are the standard deviations of the mean velocity error and density error, respectively, and $\sigma_{v}$ and $\sigma_{\rho}$ are the standard deviations of the measured mean speed and density. Conceptually, the simplest method to sensitivity analysis is to repeatedly vary one parameter at a time while holding the others fixed. A sensitivity ranking can be obtained quickly by increasing each parameter by a given percentage, while leaving all the others constant and quantifying the change in the model output. This type of analysis has been referred to as a "local" sensitivity analysis, since it addresses only sensitivity relative to the point estimates chosen and not for the entire parameter distribution [40]. The parameters of the model are changed about the nominal parameter set $\bar{U}$, defined in Table 1 , one at a time within the range $0.8 \bar{\phi}_{i}<\emptyset_{i}<1.2 \bar{\phi}_{i}$ while all other parameters are kept at their nominal (identified) values. Herein we consider the normalized deviation in the cost function $U$ from its nominal value, which is the most direct and linear way to quantify the changes [2]:

$$
\% \text { change }=\frac{(U(\varnothing)-U(\bar{\varnothing}))}{J(\bar{\varnothing})} \times 100 .
$$

In Fig. 15, the percentage of changes for each parameter are plotted, calculated by that equation. Note that EKF is more sensitive to changes in $v_{\mathrm{f}}, \tau, \delta$ and $\kappa$ compared to STSM. In contrast, STSM is more sensitive to changes in $\eta, \rho_{\mathrm{c}}$ and $a$ compared to EKF.
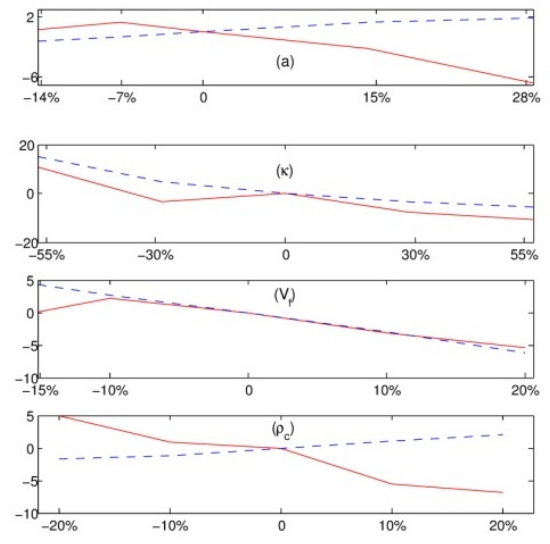
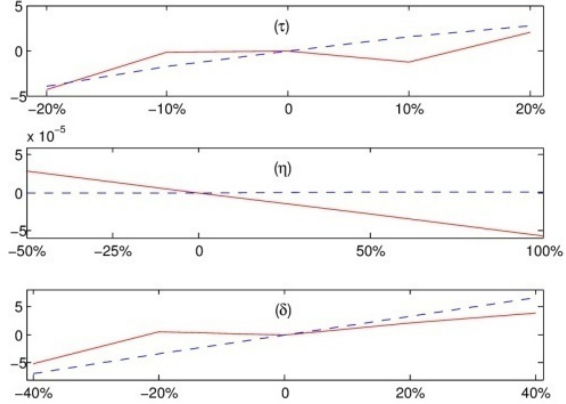

Fig. 15. Parameter sensitivity, STSM “_”, EKF “- - -” 


\section{Conclusion}

This paper presents a comprehensive comparison of two kinds of observers: STSM and EKF. The simulations are established with simulated and real data. The real data are collected on M42 motorway in the United Kingdom. For the simulation, a second order traffic flow model METANET has been used. The STSM, which behaves like a full order observer, and the structure of EKF have been presented. The following conclusions can be stated:

STSM can perform as well as EKF. The robustness of the STSM to external noise can be guaranteed. In contrast, the EKF should be tuned with respect to the external noise. It means that EKF is not robust with respect to the external noise, for accurate values of the input and measurement noise covariance matrices $Q$ and $R$ are required.

The performance of EKF in the sense of convergence and stability is better than STSM when the covariance matrices $(Q$ and $R)$ are well tuned. STSM suffers from the chattering behavior. However, STSM can steer satisfactorily the system.

STSM is the best choice compared to the EKF, since the estimation results are comparable to EKF, especially, where a small time step is taken. It is much simpler to implement, the dynamic performance can be altered and no knowledge of the noise statistics is required.

\section{References}

1. S u n, X., L. Munoz, R. Hrow it z. Mixture Kalman Filter Based Highway Congestion Mode and Vehicle Density Estimator and its Application. - In: Proc. of American Control Conference, 2004, pp. 2098-2103.

2. Kohan, R. R. Robust State Estimation and Control of Highway Traffic Systems. Toronto, Electrical and Computer Engineering, University of Toronto, 2001.

3. Majid, H., H. Abou a is sa, D. Jolly, G. Morvan. State Reconstructor for Real-Time Freeway Ramp Metering. - In: ICNSC, 2013, pp. 306-311.

4. Canudas de Wit, C., J. J. E. S l ot ine. Sliding Observers in Robot Manipulators. Automatica, Vol. 27, 1991, pp. 859-864.

5. H e r n a n d e z, J., J.-P. B a r b o t. Sliding Observer-Based Feedback Control for Flexible Joints Manipulator. - Automatica, Vol. 32, 1996, pp. 1243-1254.

6. Ma na manni, N., M. D j e mai, T. B o ukhobza, N. M'S i ridi. Nonlinear Sliding Observer Based Control for a Pneumatic Robot Leg. - Int. J. of Robotics and Automation, Vol. 16, 2001, pp. 100-112.

7. Utkin, V. I., J. Guldner, J. Shi. Sliding Mode Control in Electromechanical Systems. London, Taylor and Francis, 1999.

8. D j e m a i, M., J.-P. B a r b o t, A. G lu m i n e a u, R. B o i s live a u. Non-Linear Flux Sliding Mode Observer. - In: Proc. of IEEE CSCC99, IMACS, Athens, Greece, 1999.

9. Martinez-Guerra, R., R. Aguilar-Lo pez, A. Poznyak. Robust Sliding Mode Observers for On-Line Reaction Heat Monitoring in Continuous Chemical Reactors. ASME Journal of Dynamics Systems, Measurment and Control, Vol. 126, 2004, pp. 473-478.

10. Kn a p p, C. H. Traffic Estimation and Density Estimation for Single and Multi-Lane Traffic. Transportation Science, Vol. 7, 1973, pp. 75-84.

11. C h r i s, M., J. T q m p è r e, L. H. I m m e r s. An Extended Kalman Filter Application for Traffic State Estimation Using CTM with Implicit Mode Switching and Dynamic Parameters. 2007. 
12. Sun, X., L. Munoz, R. Horowitz. Highway Traffic State Estimation Using Improved Mixture Kalman Filters for Effective Ramp Metering Control. - In: Proc. of Conference on Decision and Control, 2003.

13. Wang, Y., M. Papa g e orgi o u. Real-Time Freeway Traffic State Estimation Based on Extended Kalman Filter: A General Approach. - Transportation Research Part B, Vol. 39, 2005, pp. 141-167.

14. Hegyi, A., D. Girimonte, R. Babuska, B. de Schutter. A Comparison of Filter Configurations for Freeway Traffic State Estimation. - In: Proc. of 2006 IEEE Intelligent Transportation Systems Conference (ITSC'2006), Toronto, Canada, 2006, pp. 1029-1034.

15. Lighthill, M. J., G. B. Whitham. On Kinematic Waves II: A Theory of Traffic Flow in Long Crowded Roads. - Proc. Royal Soc. London, Vol. A-229, 1955, pp. 317-345.

16. R i c h a r d s, P. I. Shock Waves on the Highway. - Operations Research, Vol. 4, 1956, pp. 42-51.

17. M a y, A. D. Traffic Flow Fundamentals. Englewood Cliffs, Prentice Hall, NJ, 1990.

18. P a y n e, H. J. Models of Freeway Traffic and Control. - In: G. A. Bekey, Ed. Mathematical Models of Public Systems. Vol. 1. Simulation Council Procceedings Series. La Jolia California, 1971, pp. 51-61.

19. M e s s e m e r, A., M. P a p a g o r g i o u. METANET: A Macroscopic Simulation Program for Motorway Networks. - Traffic Engineering and Control, Vol. 31, 1990, No 9, pp. 446-470.

20. Messner, A., M. P a p a g orgi o u. METANET - A Simulation Program for Motorway Networks. -Traffic Engineering and Control, Vol. 31, 1990, No 8-9, pp. 466-470.

21. L a mo n, F. Freeway Traffic Modeling and Calibration for the Eindhoven Network. Master's Thesis, Delft University of Technology. Delft Center for Systems and Control, 2008.

22. Fillipov, A. F. Differential Equaitons with Discontinous Right-Hand Side. - American Mathematics Society Transactions, Vol. 62, 1960, pp. 199-231.

23. F l o q u e t, T. Contributions à la commande par modes glissants d'ordre supèrieures. PhD Thesis, Université des Sciences et Techniques de Lille, 2000.

24. Rolink, M., T. B oukhobza, D. S a uter. High Order Sliding Mode Observer for Fault Actuator Estimation and Its Application to the Three Tanks Benchmark. In Author Manuscript, 2006.

25. S o lva r, S., V. L e, M. G h a n e s, J.-P. B a r b o t, G. S a n t o m e n n a. Observateur à mode glissant d'ordre 2 pour la machine asynchrone sans capteur mécanique. In Author Manuscript, Published in CIFA, Nancy, France, 2010.

26. S a l g a d o, I., I. Ch a ir e z, J. M or en o, L. Frid m a n, A. P o z n y a k. Generalized SuperTwisting Observer for Non Linear Systems. - In: Preprints of the 18th IFAC World Congress Milano (Italy), 28 August-2 September 2011, pp. 14353-14358.

27. Rabhi, A., N. K. M'S irdi, M. Oulad sine, L. Fridman. Estimation of Road Profile Using Second Order Sliding Mode Observer. In Authour Manuscript, 2010.

28. F r i d m a n, L., A. L e v a n t. Sliding Modes of Higher Order as a Natural Phenomenon in Control Theory. Technical Report. - In: Robust Control via Variable Structure and Lyapunov Techniques. Lecture Notes in Control and Information Science, Vol. 217. F. Garofalo, L. Glielmo, Eds. London, Springer Verlag, 1996, pp. 107-133.

29. D j e ma i, M., N. Man a mani, J.-P. B a r b ot. Sliding Mode Observer for Triangular Input Hybrid System. - In: Proc. of IFAC World Congress Prague, 2005.

30. Floquet, T., J.-P. B a rbot. Super Twisting Algorithm Based Step-By-Step Sliding Mode Observers for Nonlinear Systems with Unknown Inputs. - International Journal of Systems Science, Vol. 38, 2007, No 10, pp. 803-815.

31. D a vil a, J., L. Frid m a n, A. L e va n t. Second-Order Sliding Mode Observer for Mechanical Systems. - IEEE Transactions on Automatic Control, Vol. 50, 2005, No 11, pp. 1785-1789.

32. M'S ir di, N. K., A. R a b hi, L. Fri d m a n, J. D a vil a, Y. D e l a n n e. Second Order Sliding Mode Observer for Estimation of Velocities, Wheel Sleep, Radius and Stiffness. - Int. J. Vehicle Design, Vol. 48, 2008, No 3/4.

33. S a d a ou i, H., M. D j e mai, N. Ma namanni, K. B e man s our. Super Twisting Algorithm Observer for a Class of Switched Chaotic Systems. In Authour Manuscript, 2010.

34. M'S i rd i, N. K., A. R h b h i, L. Frid m a n, J. D a vi l a, Y. D e l a n n e. Second Order SlidingMode Observer for Estimation of Vehicle Dynamic Parameters. - Int. J. Vehicule Design, Vol. 48, 2008, No 3/4, pp. 190-207. 
35. B u i s s o n, C., J. L e s o r t. Comprendre le trafic routier Méthodes et Calculs. Certu, 2010.

36. Ch en, F., M. W. D unnigan. Comparative Study of a Sliding-Mode Observer and Kalman Filters for Full State Estimation in an Induction Machine. - IEE Proc. Electr. Power Appl., Vol. 149, 2002, No 1, pp. 53-64.

37. P a p a g e org i o u, M., H. H a d j-S a l e m, J. B l o s s e vill e. Alinea: A Local Feedback Control Law for On-Ramp Metering. Transportation Research Record, Vol. 1320, 1991, pp. 58-64.

38. P a p a g e o r g i o u, M., H. H a d j-S a l e m, F. M i d d l e h a m. Alinea Local Ramp Metering : Summary of Fields Results. - Transportation Research Record, Vol. 1603, 1997, pp. 90-98.

39. B a r r e t o, H., F. M. H o w l a n d. Introductory Econometrics Using Monte Carlo Simulation with Microsoft Excel. Cambridge, 2006.

40. Ha m b y, D. M. A Review of Techniques for Parameter Sensitivity Analysis of Environmental Models. - Environmental Monitoring and Assessment, Vol. 32, 1994, pp. 135-154.

41. Majid, H., H. Abouaissa, D. Jolly, G. Morvan. A Reduced Order Observer for Switching-Mode Model State Estimation. - In: Proc. of International Conference on Control, Decision and Information Technologies (CoDIT), Hammamet, 2013.

42. $\mathrm{Maj}$ id, H. Gestion du trafic autoroutier: contribution à l'estimation et à la commande des systèmes de transport intelligents. Presses Académiques Francophones, 2015. 